\title{
Bewertung von Sourcing- Modellen in der Bankenbranche
}

Rainer Alt/Thomas Zerndt

\section{Motivation}

Die Gestaltung der Wertschöpfungskette zählt zu den klassischen unternehmensstrategischen Aufgaben. Sie bestimmt, welche internen und externen Kompetenzen in einer Marktleistung konvergieren und welche Unternehmensform - von marktlichen „arm's length“-Beziehungen hin $\mathrm{zu}$ netzwerkartigen oder internen bzw. hierarchischen Strukturen - dabei zum Einsatz kommt. Das strategische Controlling trägt hier seit längerem zur Analyse von Eigenfertigung und Fremdbezug bei. Durch derartige Kostenvergleiche lässt sich die ökonomische Sinnhaftigkeit verschiedener Konfigurationen der Wertschöpfungskette ermitteln. Beispielsweise fließen bei vielen Schweizer Banken die durchschnittlichen Kosteneinsparungen eines Outsourcing-Projektes von 20 bis 30 Prozent gegenüber der Eigenfertigung als (Mindest)Anforderung in den Entscheidungsprozess ein (vgl. IMG 2004). Allerdings zeigen zahlreiche nicht erfolgreiche Externalisierungs-, Akquisitions- oder Fusionsprojekte die Relativität dieser anvisierten Kostenvorteile.

Die Verringerung des vertikalen Integrationsgrades gilt als ein Instrument zur Erhöhung der Anpassungsfähigkeit eines Unternehmens an interne oder externe Veränderungen (Ihde 1988, S. 19). Während Elektronik- oder Automobilunternehmen dazu verschiedene Strategien im
Supply Chain Management einsetzen (vgl. Corsten/Gabriel 2004), besitzen Banken trotz erfolgreicher Kooperationsprojekte wie etwa SWIFT im internationalen Zahlungsverkehr, noch eine beträchtliche vertikale Integration. Eine jüngst durchgeführte Befragung von 63 Geschäftsleitungsmitgliedern aus Banken im deutschsprachigen Raum deutet auf den starken "Misfit" von Eigenfertigung und Kernkompetenz hin (vgl. Falkenberg et al. 2006): Obgleich sich die meisten Banken nach Eigeneinschätzung über ihre Vertriebs- und Beratungskompetenz klar differenzieren, dominiert gleichzeitig in fast allen Prozessen die Eigenfertigung. Zahlreiche neue Organisationsformen in der Banken-Wertschöpfungskette zeigen eine zunehmende Bereitschaft zur Externalisierung von Zahlungsverkehr-, Wertpapierund teilweise auch der Kreditabwicklung und deuten auf die sukzessive Annäherung der realen Eigenfertigungsstruktur an das bereits heute formulierte Kernkompetenzprofil hin. Banken wie die Zürcher Privatbank Maerki Baumann oder die Bank Vontobel bieten mit ihren Insourcing-Strategien bereits anderen Banken Leistungen z. B. zur Wertpapierabwicklung an. Ebenso weiten etablierte Dienstleister im Schweizer Bankenbereich wie etwa BSource, RBA-Service oder Sourcag ihre Marktleistungen aus. Diese Entwicklungen finden zunehmend auch grenzüberschreitend statt.
- Sourcing-Modelle bestimmen die Organisation der Wertschöpfungsaktivitäten und werden u. a. von Banken zur vertikalen Desintegration diskutiert.

- Die Eignung eines SourcingModells ist vor allem situativ begründet; ein pauschal bestgeeignetes Sourcing-Modell existiert nicht.

- Entscheider benötigen Instrumente zur Ermittlung geeigneter Sourcing-Modelle. Hier liefern die Ansätze des strategischen Controllings einen ersten Beitrag. - Zur ganzheitlichen, standardisierten, transparenten und nachvollziehbaren Bewertung für Banken sind die bestehenden Ansätze zu kombinieren und zu erweitern. - Jede Bewertung benötigt eine vorhergehende (Grob-)Modellierung; für eine simultane Modellierung und Bewertung fehlen noch integrierte Verfahren.

\section{Grundlagen und Anforderungen}

Der vorliegende Beitrag bezeichnet die Gestaltung der Wertschöpfungskette neudeutsch als „Sourcing“. Im Logistikbereich wird darunter seit längerem ein Teil der Beschaffungsstrategie verstanden, wel-

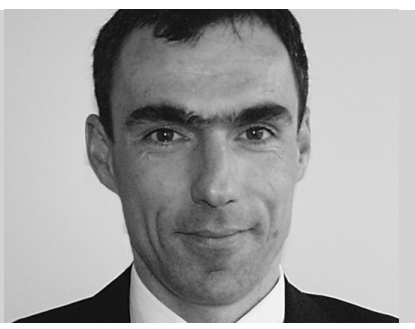

\section{Dr. Rainer Alt}

ist Professor für Wirtschaftsinformatik, insbesondere Anwendungssysteme in Wirtschaft und Verwaltung an der Universität Leipzig (www.iwi.uni-leipzig.de) und hat gemeinsam mit Thomas Zerndt am Institut für Wirtschaftsinformatik der Universität St. Gallen, Schweiz das Kompetenzzentrum Sourcing in der Finanzindustrie (sourcing.iwi.unisg.ch) gegründet. 
che das Unternehmen mit seinen vorgelagerten Versorgungsmärkten verbindet (Klaus/Krieger 2004, S. 54). Eine Weiterentwicklung versteht unter Sourcing die Neuausrichtung der gesamten Wertschöpfungskette, wobei jedes Unternehmen seine Kernkompetenzen wahrnimmt und für die übrigen Aktivitäten auf Partnerressourcen zurückgreift (Gottfredson et al. 2005, S. 151). Um begriffliche Unschärfen zu vermeiden, wird Sourcing nachfolgend als die Gestaltung und Realisierung arbeitsteiliger Kunden-Lieferanten-Beziehungen verstanden, unabhängig, ob es sich dabei um interne oder externe Leistungserbringer handelt. Die bekanntesten SourcingStrategien lassen sich anhand der Richtung des Leistungsbezugs beschreiben: beim Outsourcing verlagert ein Unternehmen bzw. Outsourcer einen Geschäftsprozess insgesamt oder partiell an einen Dienstleister bzw. Insourcer. Während beim Insourcing ein Unternehmen seine Unternehmensgrenze erweitert, findet beim Outsourcing eine Reduktion des eigenen Wertschöpfungsumfanges statt. In Anlehnung an ein Sourcing-Konzept, das „die kleinste Einheit einer Beschaffungslogistik- bzw. Beschaffungsstrategie“ darstellt (Klaus/Krieger 2004, S. 476), bezeichnet ein Sourcing-Modell hier die konkrete Ausgestaltung einer Sourcing-Strategie für einen Geschäftsprozess.

Innerhalb des Controlling befasst sich vor allem das strategische Controlling mit der Gestaltung von Sourcing-Modellen. Gegenüber dem operativen Controlling, das eine kurz- bis mittelfristige Perspektive auf die laufenden Prozesse im Rahmen der jeweils für die Organisationseinheit gegebenen Ziele einnimmt, betrachtet es die längerfristigen gesamtheitlichen Aspekte der Ziel- und Aufgabenentwicklung und der Erfolgskontrol- le einer Organisationseinheit (vgl. Stahl 1992). Aus überbetrieblicher Sicht versucht das strategische Controlling, die Ursachen der geringen Erfolgsquote von Netzwerkprojekten und Verbesserungsmöglichkeiten zu identifizieren (Jehle 2003, S. 379). In ähnlicher Weise betrachtet es (Möller 2002, S. 97) als Aufgabe des Controlling, „stringente und schlüssige Gestaltungsvorschläge “ für Koordinationsaufgaben von Marktpartnern zu entwickeln. Das Netzwerkcontrolling (Hess 2002, S. 94) konkretisiert diese Aufgaben grob in Netzwerkkonfiguration und -ausführung bzw. eine auftragsübergreifende und -bezogene Ebene.

Auf dieser Basis lässt sich die Entscheidung über Sourcing-Modelle als Unterstützung bei der Netzwerkkonfiguration begreifen. Aufgrund der typischerweise unstrukturierten Entscheidungssituationen bedient sich das strategische Controlling weniger der Optimierungsmodelle des Operations Research, sondern vielmehr entscheidungstheoretischer Auswahl- und Bewertungsmodelle (Klein/ Scholl 2004, S. 37). Wie bereits aus der Erfolgsfaktorenforschung bekannt ist, verhindert die Vielschichtigkeit der unternehmerischen Realität klare deterministische Zusammenhänge, was eine Ermittlung der Kausalität zwischen ,optimaler“ Handlung und erfolgreicher Wirkung verunmöglicht. Zahlreiche Erfolgsfaktorstudien konnten nicht einmal eine nachhaltige Wirkung des Marketings auf den Unternehmenserfolg belegen (Fritz 1990). Ziel ist daher die Ermittlung einer situativ „optimalen“ Wertschöpfungskettenkonfiguration - nicht pauschales maximales Outsourcing steht daher im Vordergrund, sondern die Bestimmung des geeigneten Sourcing-Modells für ein spezifisches Unternehmen (vgl. Dichtl 1991).
Zur Beurteilung von Sourcing-Vorhaben wendet die Unternehmenspraxis unterschiedliche Techniken an, die auf eine „Entsubjektivierung“ anhand bestimmter vorgegebener und nachvollziehbarer Maßstäbe gerichtet sind. Deshalb wird im vorliegenden Beitrag auch von einem Bewertungs- und nicht von einem Beurteilungsmodell gesprochen (Wikipedia 2006). Während diese Frage eher akademischer Natur ist, steht für die Unternehmenspraxis die Beantwortung folgender Fragen im Vordergrund:

- Führt das Outsourcing zu Einsparungen gegenüber der Ausgangssituation von mindestens 20 Prozent? Diese quantitative Forderung geht von den (häufig nicht vorhandenen) Ist-Kosten eines Unternehmens oder eines Bereiches aus und zeigt periodenbezogene Kosteneffekte für ein Soll-Modell.

- Welches sind die zeitraumbezogenen Kostenwirkungen unter Berücksichtigung pessimistischer, wahrscheinlicher und optimistischer Rahmenbedingungen, z. B. bei Annahme bestimmter Transaktionsvolumina? Business Cases mit Bandbreitenbetrachtungen („Bestcase/Worstcase“) erlauben es beispielsweise, Marktentwicklungen bezüglich eines Sourcing-Modells zu simulieren.

- Welche Auswirkungen hat das Sourcing-Projekt auf die Cost-IncomeRatio, also das Verhältnis von operativem Aufwand und operativem Ertrag als klassischem Effizienzmaßstab von Bankinstituten? Elemente von Business Cases sowie der Kostenrechnung fließen in dieser Kennzahl zusammen, die abhängig vom Banktyp von ca. 45 Prozent bis über 70 Prozent reicht (Dang/Lau 2006, S. 23).

- Mit welchen strategischen Potenzialen und Risiken ist das Sourcing verbunden? Dazu zählen der Übergang vom kostenbestimmten Sourcing hin zu einem „Strategic Sourcing“ (vgl. Welch/Nayak 1992, Gottfredson et al. 2005) und die Berücksichtigung der verstärkten Abhängigkeit von einem nicht direkt kontrollierbaren Partner (Principal-Agent-Problematik) (vgl. Hess 2002, S. 94, Keil 2005). 
- Welche Maßnahmen sind zur Steuerung des zukünftigen Sourcing-Modells notwendig? Fragen bezüglich Verantwortlichkeiten und Vereinbarungen zur Ausgestaltung der künftigen Kooperation und der Governance in der Sourcing-Beziehung sind hier zu lösen. Auch die Bewertung schwer messbarer Faktoren wie etwa des Vertrauens in einen Partner ist zu adressieren.

- Welche partnerspezifischen Anpassungen sind zur Umsetzung des SourcingModells notwendig? Hierbei kann es sich ebenso um die Definition von Zielgrößen bzw. „Service Levels“ wie um Schnittstellenanpassungen bei den Informationssystemen (IS) handeln.

Diese Fragen illustrieren die Heterogenität der Einflussfaktoren bei der Wahl eines Sourcing-Modells und führen zu verschiedenen Anforderungen an die Bewertung von Sourcing-Modellen (s. Tabelle 1). Offensichtlich kommen allgemeine entscheidungstheoretische Techniken wie etwa die Nutzwertanalyse zwar zum Einsatz, jedoch geben diese keine inhaltlichen Kriterien vor. Hier setzen die nachfolgend beschriebenen quantitativen und qualitativen Bewertungsansätze an.

\section{Analyse bestehender Bewertungsansätze}

Zur Beurteilung der Leistungstiefe lassen sich grob drei theoretische Richtungen unterscheiden (vgl. Picot 1991, S. 340 ff., Schildhauer 2001, S. 71): qualitativunternehmenspolitische, transaktionskostentheoretische und quantitativ-kostenrechnerische Ansätze sowie der Transaktionskostenansatz. Etwas vereinfachend beschreibt dieser Beitrag quantitative, qualitative und kombinierte Ansätze anhand der in Tabelle 1 genannten Anforderungen.

Quantitative Ansätze sind die klassischen Verfahren zur Messung einer Sourcing-Entscheidung bezüglich ihrer Kosteneinsparungs- und Rentabilitätspotenziale. Vielfach werden dabei Marktpreise den entscheidungsrelevanten Kosten der Eigenfertigung gegenüber gestellt (Picot

Tabelle 1: Anforderungen zur Bewertung von Sourcing-Modellen bei Banken

\begin{tabular}{l|l}
\hline Kriterien & Ausprägungen \\
\hline Gestaltungsebenen & $\begin{array}{l}\text { Transformationsprojekte umfassen Veränderungen auf den Ebenen Strategie, Prozess und } \\
\text { (Informations-) System (vgl. Österle 1995). }\end{array}$ \\
\hline Sichtweise & $\begin{array}{l}\text { Die Bewertung soll die Auswirkungen auf die Marktposition, die internen Abläufe und die } \\
\text { Komplexiẗ̈t der Transformation berücksichtigen. }\end{array}$ \\
\hline Messkriterien & $\begin{array}{l}\text { Bewertungen sollen qualitativ und quantitativ erfolgen sowie eine retrospektive und prospektive } \\
\text { Sicht unterstützen. }\end{array}$ \\
\hline Umsetzbarkeit & $\begin{array}{l}\text { Die Bewertung soll einem Vorgehensmodell folgen, standardisiert und flexibel anwendbar sein } \\
\text { und mittels eines IT-Tools durchführbar sein. }\end{array}$ \\
\hline Domänenbezug & $\begin{array}{l}\text { Es soll ein klarer Bezug des Modells zur Bankenbranche, zur Value-Chain-Gestaltung und zum } \\
\text { Sourcing bestehen. }\end{array}$ \\
\hline Aussagen & $\begin{array}{l}\text { Eine möglichst ganzheitliche Bewertung soll Kosten, Nutzen, Potenziale sowie eine Risiko- } \\
\text { bewertung einschließen. }\end{array}$ \\
\hline
\end{tabular}

1991, S. 340f). Während Erstere durch Angebotseinholung verhältnismäßig einfach zu ermitteln sind, bestehen bei den Eigenfertigungskosten häufig erhebliche Probleme: entweder sie basieren auf reinen Annahmen oder sie vernachlässigen die im Bankenbereich signifikanten Gemeinkosten (Kipker/Veil 2003, S. 193). Eine Folge ist die Fehleinschätzung der Eigenfertigungseffizienz, wie sie auch eine Studie von „Business Process Outsourcing"-Projekten (BPO) bei 91 Banken zeigt: Während gerade 13 Prozent der Befragten ohne Outsourcing-Erfahrung an skalenbedingte Kostenvorteile glaubten, waren dies 64,3 Prozent der BPO-erfahrenen Banken (Beimborn et al. 2005, S. 436). Eine weitgehend auf Kostenschlüsselung und -verrechnung beruhende Kostenträgerrechnung gilt daher für produktionstopologische Entscheidungen als ungeeignet (Bernet 1998, S. 34). Eine Erweiterung liefert die Prozesskostenrechnung zur aktivitätsorientierten Zuordnung von Gemeinkosten durch verrechnungsfähige Umlagegrößen (Nadig 2000, S. 14). Damit lassen sich die Eigenfertigungskosten für auszulagernde Aktivitäten, wie etwa die Auftragserfassung oder -abwicklung ebenso wie die produktbezogene Kostenzurechnung, also etwa für eine Überweisung oder die Abwicklung einer Wertpapierorder, ermitteln. Die „Total Cost of Ownership“-
Methode schliesslich geht darüber hinaus und versucht eine Beurteilung der Gesamtkosten einer Investitionsentscheidung, also einschließlich der einer Sourcing-Entscheidung vor- und nachgelagerten Kosten. So sind beim Outsourcing im Wertpapierbereich neben den Prozesskosten (Stückkosten des WertpapierServiceproviders) auch der Steuerungsaufwand (Kosten für verbleibende Einheiten) und die Projektkosten (Zusatzkosten durch das Outsourcing-Projekt) zu berücksichtigen (Lubich/Aumer 2003, S. 55). Zusammenfassend sind quantitative Bewertungsansätze die Basis einer Sourcing-Entscheidung, da sie die anfallenden Kosten auf Prozess- und Systemebene erfassen und gut operationalisierbar sind. Jedoch vernachlässigen sie die strategische Erlössicht ebenso wie potenzialorientierte und risikobezogene Faktoren (vgl. Simschek 2005).

Hier setzen qualitative Verfahren an, die vor allem aus dem strategischen $\mathrm{Ma}$ nagement sowie der Neuen Institutionenökonomik stammen. Erstere umfassen ressourcen- und kernkompetenzorientierte Ansätze zur Positionierung und Differenzierung im Wettbewerbsumfeld. Beispielsweise beschreiben (Hagel/Singer 1999) ausgehend von drei betrieblichen Kernressourcen die Positionierungsoptionen des problemlösungsorientierten Dienstleisters im Kundenkontakt, des 
Tabelle 2: Bewertungsverfahren im Überblick

\begin{tabular}{|c|c|c|c|c|c|}
\hline $\begin{array}{l}\text { Kriterien mit } \\
\text { Ausprägung }\end{array}$ & Kostenrechnung & $\begin{array}{c}\text { Prozesskosten- } \\
\text { rechnung }\end{array}$ & $\begin{array}{l}\text { Total Cost of } \\
\text { Ownership }\end{array}$ & $\begin{array}{l}\text { Strategisches } \\
\text { Management }\end{array}$ & $\begin{array}{l}\text { Transaktions- } \\
\text { kostentheorie }\end{array}$ \\
\hline \multicolumn{6}{|c|}{ Gestaltungsebenen } \\
\hline Strategie & $\bullet$ & 0 & $\bullet$ & $\bullet$ & $\bullet$ \\
\hline Prozess & 0 & $\bullet$ & $\bullet$ & $\Delta$ & $\Delta$ \\
\hline System & $\bullet$ & $\Delta$ & $\bullet$ & 0 & 0 \\
\hline \multicolumn{6}{|l|}{ Sichtweise } \\
\hline Aussensicht & 0 & 0 & $\bullet$ & $\bullet$ & $\bullet$ \\
\hline Innensicht & $\bullet$ & $\bullet$ & $\bullet$ & $\bullet$ & 0 \\
\hline Transformation & $\Delta$ & $\bullet$ & $\bullet$ & 0 & 0 \\
\hline \multicolumn{6}{|l|}{ Messkriterien } \\
\hline Quantitativ & $\bullet$ & $\bullet$ & $\bullet$ & 0 & 0 \\
\hline Qualitativ & 0 & 0 & $\bullet$ & $\bullet$ & $\bullet$ \\
\hline Retrospektiv & • & $\bullet$ & $\bullet$ & $\bullet$ & \\
\hline Prospektiv & 0 & 0 & $\bullet$ & 0 & $\bullet$ \\
\hline \multicolumn{6}{|l|}{ Umsetzbarkeit } \\
\hline Vorgehensmodell & $\bullet$ & $\Delta$ & $\Delta$ & 0 & 0 \\
\hline Tool-Support & $\bullet$ & $\bullet$ & $\Delta$ & 0 & 0 \\
\hline Standardisierung & $\Delta$ & $\Delta$ & 0 & 0 & 0 \\
\hline Flexibilitüt & $\Delta$ & $\Delta$ & $\bullet$ & $\bullet$ & $\bullet$ \\
\hline Verbreitung & $\bullet$ & $\bullet$ & $\bullet$ & $\Delta$ & $\Delta$ \\
\hline \multicolumn{6}{|l|}{ Domänenbezug } \\
\hline Bankenbranche & $\Delta$ & $\Delta$ & $\Delta$ & 0 & 0 \\
\hline Value Chain & 0 & $\Delta$ & $\Delta$ & 0 & 0 \\
\hline Sourcing & $\bullet$ & 0 & $\Delta$ & 0 & $\bullet$ \\
\hline \multicolumn{6}{|l|}{ Aussagen } \\
\hline Kosten & $\bullet$ & - & $\bullet$ & 0 & $\Delta$ \\
\hline Nutzen & 0 & 0 & 0 & $\bullet$ & $\Delta$ \\
\hline Potenziale & 0 & 0 & 0 & $\bullet$ & 0 \\
\hline Risiken & 0 & 0 & $\Delta$ & 0 & $\bullet$ \\
\hline \multicolumn{6}{|c|}{ Zusammenfassende Eignung } \\
\hline & $\begin{array}{l}\text { Kostenbeurteilung } \\
\text { mit Ist-/Vergan- } \\
\text { genheitsdaten }\end{array}$ & $\begin{array}{l}\text { Kostenbasierte } \\
\text { Bewertung von } \\
\text { Aktivitäten und } \\
\text { Leistungen }\end{array}$ & $\begin{array}{l}\text { Kostenorientierte } \\
\text { Bewertung } \\
\text { interner Poten- } \\
\text { ziale auf } \\
\text { taktischer Ebene }\end{array}$ & \begin{tabular}{|l|} 
Bewertung \\
internner Poten- \\
ziale auf \\
strategischer \\
Ebene
\end{tabular} & $\begin{array}{l}\text { Bewertung } \\
\text { qualitativer } \\
\text { Faktoren auf } \\
\text { strategischer } \\
\text { Ebene }\end{array}$ \\
\hline
\end{tabular}

Legende: — voll erfüllt, $\Delta$ teilweise erfüllt, $\bigcirc$ nicht erfüllt noch Prozess- und Systemaspekte berücksichtigen. Um qualitative Zusammenhänge wie strategische Bedeutung, Unsicherheit oder opportunistisches Partnerverhalten systematischer zu erfassen, wurde seit den 90er Jahren verstärkt die Neue Institutionenökonomik, insbesondere die Transaktionskostentheorie eingesetzt (vgl. Picot et al. 2002, S. 54 f.). Diese versucht die effizienten Unternehmensgrenzen zu bestimmen (Williamson 1985, S. 109 ff.), die letztlich auch zum situativ bestgeeigneten Sourcing-Modell führen. Eine Auslagerung gilt als umso sinnvoller, je weniger spezifisch eine Partnerbeziehung für das Unternehmen ist und je geringer die Risiken eines Providerwechsels einzustufen sind. Ein quantitatives Messen i.S.d. Kostenrechnung nimmt die Transaktionskostentheorie aber nicht vor (Bruch 2000, S. 20), vielmehr versucht sie qualitative Konstrukte empirisch zu erheben, etwa in Form von Fragebögen. Zusammenfassend liegt gerade in der verhältnismäßig strukturierten und nachvollziehbaren Bewertung der Beitrag qualitativer Ansätze. Sie ergänzen die quantitativen Ansätze, besitzen aber einen hohen Abstraktionsgrad und sind wenig auf einen Anwendungsbereich, z. B. die Bankenbranche, bezogen (s. Tabelle 2).

\section{Bewertungsansatz für das Financial Sourcing}

Zur Kombination und Konkretisierung der allgemeingültigen Bewertungskriterien anhand des Financial Sourcing sind multitheoretische und -dimensionale Bewertungsmodelle entstanden (vgl. Amberg et al. 2005, S. 2). Nachfolgend werden zwei bestehende Beiträge charakterisiert (vgl. Simscheck 2005) und ein dritter detaillierter entwickelt.

Das „Strategic Sourcing Framework“ von (Lammers 2004, S. 205 f.) verbindet Kriterien aus Transaktionskostentheorie und dem ressourcenbasierten Ansatz zur Eingrenzung der Sourcing-Optionen „make“, „share“ und „buy“. Es schließt sich eine quantitative Bewertung an, welche die relevanten Prozesskosten ermittelt und anhand externer Benchmarks ver- 
gleicht. Mittels der „Economies of Skill“, „Economies of Scale“ und „Economies of Scope " wird unter den Alternativen dann anhand eines mathematischen Modells die kosteneffiziente Alternative gewählt, mittels einer dynamischen Kostenrechnung über mehrere Perioden abgezinst und schließlich um eine Wertbetrachtung ergänzt. Eigenfertigung kommt nur bei gesteigertem Unternehmenswert zum Einsatz. Aus Sicht der Unternehmenspraxis dürfte das theoriegetriebene Modell allerdings nicht einfach operationalisierbar sein und mit drei generischen Sourcing-Modellen das Organisationsspektrum von Bankprozessen stark vereinfachen.

Der „IBI-Kubus“ ist ein zweites kombiniertes bankenspezifisches Modell (Locher/ Mehlau 2002, S. 17 ff.), das in einem Portfolio Ausprägungen aus der Transaktionskostentheorie („Spezifität“), des ressourcenbasierten Ansatzes („strategische Relevanz“) und der Kostenvergleichsrechnung („Produktions- und Transaktionskosten“) verbindet. Diese Kriterien gliedern sich in weitere Unterkriterien auf, werden anhand einer Nutzwertanalyse beurteilt und schließlich in einem Portfolio zur Ableitung einer Empfehlung positioniert. $\mathrm{Zu}$ sätzlich erfolgt eine Beurteilung (aufsichts-)rechtlicher Rahmenbedingungen. Wie das „Strategic Sourcing Framework“ besitzt auch der „IBI-Kubus“ einen hohen Abstraktionsgrad und mit den Normstrategien nur generische Empfehlungen zum geeigneten Sourcing-Modell. Letztlich eignet sich der IBI-Kubus eher zur Vorbereitung einer Sourcing-Entscheidung und weniger zur Auswahl eines SourcingModells selbst.

Beide Ansätze führen die Bewertung auf Basis eines bestehenden Prozessmodells durch. Es findet also keine integrierte Gestaltung und Bewertung statt, sondern vielmehr eine der Modellierung nachgelagerte. Da dieses Vorgehen auch eine hohe Praxistauglichkeit besitzt (Jehle 2003, S. 384), folgt auch das dritte darzustellende Bewertungsmodell diesem Vorgehen. Dieses ist das Ergebnis eines am Institut für Wirtschaftsinformatik der Universität St.Gallen durchgeführten Projekts mit zwölf Unternehmen aus dem

\section{Definitionen zur Beurteilung von Sourcing-Modellen}
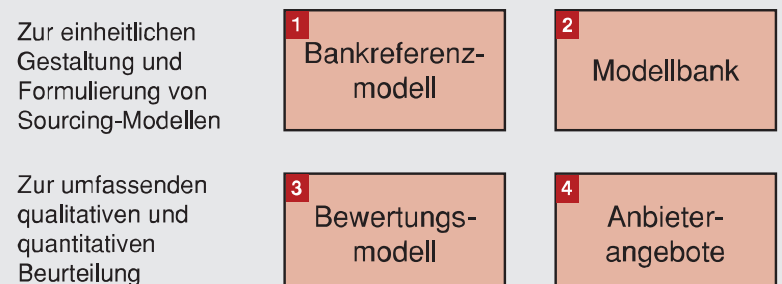

Zur Formulierung von Modellannahmen für die praxisorientierte Anwendung
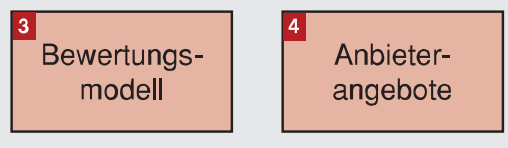

Zur praxisnahen Anwendung und Verifikation des Bewertungsmodells
Bankenbereich. Zu den wichtigsten Projektaktivitäten zählten die Definition (vgl. Abbildung 1):

1. eines Bankenreferenzmodells, um eine stabile Diskussionsgrundlage für die unterschiedlichen Prozessarten und Prozesse eines Bankbetriebs zu schaffen. Aufgrund ihres hohen Strukturierungs- und Standardisierungsgrades waren die Abwicklungsprozesse in den Bereichen Zahlungsverkehr, Wertpapier und Kredite von besonderer Sourcing-Relevanz und wurden auf drei Detaillierungsebenen anhand eines (Gesamt)Bankenreferenzmodells modelliert.

2. einer Modellbank als stabiler Bewertungsgrundlage. Das Modellbank-Prinzip folgt der eingangs formulierten Hypothese, wonach eine pauschale Eignung von Sourcing-Modellen aufgrund der zahlreichen Einflussparameter (z. B. Gesamtbankstrategie, Wettbewerbsposition, Kostenstruktur, Volumina) nicht sinnvoll ist und führt mittels bestimmter Annahmen zu einer verbesserten Kontrollierbarkeit und Nachvollziehbarkeit der Bewertung.

3. eines Bewertungsmodells, das quantitative und qualitative Kriterien in einem bankspezifisch ausdifferenzierten Kriterienkatalog zusammenstellt und damit eine standardisierte Bewertungsgrundlage von Sourcing-Modellen bereitstellt. Damit sollen Entscheidungsträger in Banken alternative Sourcing-Modelle ganzheitlich bezüglich ihrer Potenziale und Risiken vergleichen können.

4. realistischer Anbietergebote für die anhand des Bankenreferenzmodells definierten Sourcing-Modelle der Modellbank. Die Eignung dieser konkreten Angebote wird anhand des Bewertungsmodells ermittelt und führt zur Bestimmung des „optimalen“ Sourcing-Modells für die Modellbank.

Diese vier Elemente wirken in einem Vorgehen zur Gestaltung von Sourcing-Modellen (s. Abbildung 2) zusammen. Ausgangspunkt ist die übergreifende Bankstrategie und auf den Ebenen Strategie, Prozess und IS grob modellierte SourcingModelle. Bei Referenzmodellen sind diese Alternativen bereits vordefiniert und damit unmittelbar anwendbar. Zur Formulierung ihrer Sourcing-Strategie folgen Unternehmen der allgemeinen Marktpositionierung und Ressourcenpriorisierung wie sie aus der Unternehmensstrategie hervorgehen. Damit beschreibt die SourcingStrategie die grundsätzliche Form der Leistungserstellung für die Gesamtbank und macht Vorgaben zur Ausgestaltung von Sourcing-Modellen. In der Vorselektion erfolgt die prozessbezogene Konkretisierung für Vertriebs-, Abwicklungs-, Leistungs- und Unterstützungsprozesse anhand vorgegebener Sourcing-Modelle, welche jeweils gebündelte Aktivitäten aus den Referenzprozessen darstellen. Die Selektion ergibt sich aus der Gegenüberstellung dieser Modelleigenschaften mit den Eigenschaften vordefinierter Banktypen, also etwa den Rahmenbedingungen einer idealtypischen Retailbank. Eine genauere Bewertungsgrundlage ergibt sich jedoch erst mit Ausgestaltung eines oder mehrerer Sourcing-Modelle von einem entsprechenden Anbieter bzw. Insourcer. Das Bewertungsmodell ermittelt schließlich aus 
mehreren Alternativen anhand gleich bleibender Kriterien das bevorzugte bzw. situativ „optimale“ Sourcing-Modell.

Gemäß der Vorgaben bildet das Bewertungsmodell Sourcing-Empfehlungen umfassend ab. Die qualitative Bewertung besteht aus einem Katalog aus 130 Kriterien, die aus den oben erwähnten theoretischen qualitativen Ansätzen abgeleitet, in sechs Klassen nach (Pohland 2000) gegliedert und von den Forschungspartnern ergänzt wurden (s. Tab. 3). Eine enge Verzahnung mit dem Gestaltungsmodell (Prozess- und IS-Modell) findet sich bei der Ermittlung von Prozess- und Funktionsabdeckung, die einen Erfüllungsgrad je modelliertem Prozessschritt bzw. Systemfunktionalität vorsieht und mit dem Bezug zum Bankmodell eine konsistente Prozessbeurteilung unterstützt. Aus den gewichteten Nutzwerten der Einzelkriterien resultiert mit dem Gesamtnutzwert das qualitative Eignungsprofil einer Sourcing-Alternative, das sich z. B. in Form eines mehrdimensionalen Netzdiagramms gemeinsam mit einem Anforderungsprofil visualisieren lässt.

Ein ähnliches Vorgehen führt zur Ermittlung der quantitativen Bewertungsinstrumente. Aus der Verbindung von Kosten- und Investitionskostenrechnung bildet eine Business Case-Analyse die Fixkos- ten (Personal, Hard- und Software), bankproduktspezifische variable Kosten (z. B. Kosten je Einzel- oder Dauerauftrag) sowie einmalige und wiederkehrende Erträge jeweils kalkuliert über mehrere Perioden (z. B. einen Zeitraum von zehn Jahren) ab und kalkuliert den Kapitalwert eines bestimmten Sourcing-Modells. Mittels unterschiedlich angenommener Rationalisierungseffekte und Erlöswirkungen lassen sich pessimistische bis optimistische Szenarios untersuchen. Schließlich stellt die Erfolgsrechnung operative Überschüsse und Kosten gegenüber und erlaubt damit eine Annäherung an die CIR und deren Entwicklung über die nächsten Perioden (s. Tabelle 4).

\section{Anwendungsbeispiel}

Eine realitätsnahe Anwendung des Bewertungsmodells haben Vertreter von zwölf Unternehmen aus dem Bankenbereich am Beispiel der Modellbank vorgenommen. Diese repräsentiert eine Schweizer Universalbank mit einem starken Privatbank-Bereich, die aufgrund ihrer Positionierung als Vertriebsbank die Abwicklung von Zahlungs-, Wertpapier- und Kredittransaktionen sowie die Kunden-, Konto- und Depotführung als grundsätzlich externalisier- bar beurteilt hat. Ziel ist es, durch Rekrutierung sowie Schulung zusätzlicher Kundenberater den strategischen Fokus zu stärken und mittels Sourcing-Maßnahmen die CIR zu verbessern. Letztlich sollen Kostenreduktionen einen Ausbau des bestehenden Filialnetzes unterstützen. Während ein externer Dienstleister bereits vollständig die Zahlungsverkehrsabwicklung übernommen hat, werden für die übrigen Abwicklungsbereiche Angebote vom Markt eingeholt. Das vorliegende Beispiel beschränkt sich auf den Wertpapierbereich, in welchem die Modellbank gegenwärtig ein umfangreiches Korrespondenzbankenund Brokernetzwerk sowie elektronische Verbindungen zu mehreren internationalen Börsenplätzen unterhält. Die Vorselektion führte zu drei Sourcing-Modellen für die Wertpapierabwicklung:

- Modell 1 betrifft die Fremdvergabe des Auftragshandels mit Leitwegebestimmung, Pooling im Partnernetzwerk und Platzierung von Börsenaufträgen.

- Modell 2 betrifft mit der Auftragsabwicklung die gesamte Auslagerung des Backoffice, u.a. Spesen- und Gebührenermittlung, Verbuchung und Kundenoutput.

- Modell 3 betrifft die vollständige

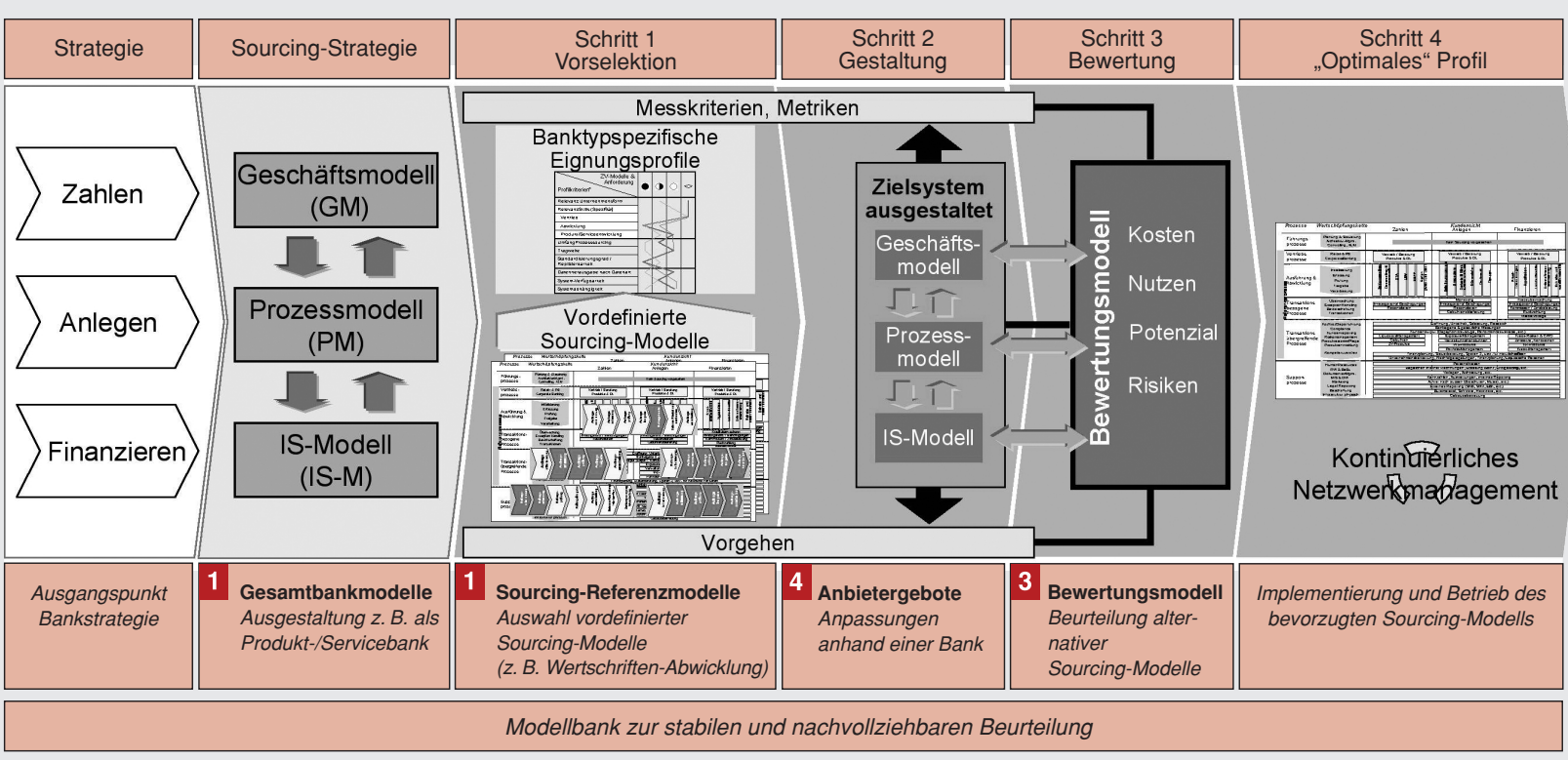

Abbildung 2: Vorgehen bei der Bestimmung des, optimalen' Sourcing-Modells 
Tabelle 3: Qualitativer Kriterienkatalog des Bewertungsmodells

\begin{tabular}{l|l|l}
\hline Kriterienklassen & Kriterien Ebene 1 & Kriterien Ebene 2 (Auszug) \\
\hline $\begin{array}{l}\text { Geschäfts- und } \\
\text { Vernetzungstreiber }\end{array}$ & $\begin{array}{l}\text { Strategische Handlungsoption } \\
\text { Governance in der Partnerschaft } \\
\text { Community } \\
\text { Komplexitätsmanagement }\end{array}$ & $\begin{array}{l}\text { Strategische Bedeutung, Einfluss auf Kernkompetenz, } \\
\text { Vertrauen in Partner, kultureller Fit, } \\
\text { Dominanz in der Community, Anzahl Partner }\end{array}$ \\
\hline Politiktreiber & $\begin{array}{l}\text { Regulatorien } \\
\text { Offentlichkeit } \\
\text { Stakeholder }\end{array}$ & $\begin{array}{l}\text { Erfüllung nationaler und internationaler Regulatorien, } \\
\text { Akzeptanz in der Öffentlichkeit, } \\
\text { Kundenakzeptanz }\end{array}$ \\
\hline Kostentreiber & $\begin{array}{l}\text { Mengen } \\
\text { Prozessbeurteilung } \\
\text { Prozessabdeckung }\end{array}$ & $\begin{array}{l}\text { Margenveränderung absolut, kritische Größe } \\
\text { Transaktionsvolumen, } \\
\text { Standardisierungsgrad, Prozesslücken }\end{array}$ \\
\hline $\begin{array}{l}\text { System- und } \\
\text { Techniktreiber }\end{array}$ & $\begin{array}{l}\text { Systembeurteilung qualitativ } \\
\text { Funktionsabdeckung }\end{array}$ & $\begin{array}{l}\text { Mandantenfähigkeit, Skalierbarkeit, Datensicherheit, } \\
\text { Funktionslücken }\end{array}$ \\
\hline Risikotreiber & $\begin{array}{l}\text { Finanzielles Risiko } \\
\text { Marktrisiko } \\
\text { Operationelles Risiko } \\
\text { Partnerschaftsrisiko } \\
\text { Personelles Risiko }\end{array}$ & $\begin{array}{l}\text { Monetäre Verluste, Konkurrenzrisiko, Reputationsverlust, } \\
\text { Teilen von Geschäfts- und Kundendaten, } \\
\text { Konzentrationsrisiko, Gegenparteirisiko, } \\
\text { Motivation Mitarbeiter, Know-how Verlust }\end{array}$ \\
\hline Transformationstreiber & $\begin{array}{l}\text { Projekt - Planung } \\
\text { Projekt - Umsetzung } \\
\text { Projekt - Kontrolle und Steuerung } \\
\text { Change }\end{array}$ & $\begin{array}{l}\text { Zeitaufwand, Ressourcenverfügbarkeit, } \\
\text { Projektkomplexität, Anpassungsfähigkeit, Kosten- } \\
\text { tau-/Ablauforganisation, Projekterfahrung des Partners }\end{array}$ \\
\hline
\end{tabular}

Tabelle 4: Zusammenfassung der quantitativen Effekte in der CIR (Ausschnitt für 2 Perioden)

\begin{tabular}{|c|c|c|c|c|c|}
\hline & IST $(t=0)$ & $\Delta$ Jahr 1 & SOLL $(t=1)$ & $\Delta$ Jahr 2 & SOLL ( $t=2)$ \\
\hline Zinsüberschuss & 29.024 & & 29.024 & & 29.024 \\
\hline Provisionsüberschuss & 78.773 & & 78.773 & & 78.773 \\
\hline Verwaltungskosten, davon & -57.565 & 10.354 & -47.211 & 11.564 & -46.001 \\
\hline Personalkosten & -39.161 & 7.800 & -31.361 & 7.800 & -31.361 \\
\hline Sachkosten & -16.493 & 7.000 & -9.493 & 7.000 & -9.493 \\
\hline Abschreibungen auf Sachanlagen & -1.610 & 0 & -1.610 & 0 & -1.610 \\
\hline Sonstiges (Stevern, Lizenzen, Sourcing) & -301 & 1.300 & 999 & 1.300 & 999 \\
\hline Sourcing- Kosten & & -5.746 & -5.746 & -4.536 & -4.536 \\
\hline Teilbetriebsergebnis & 50.232 & & 60.586 & & 61.796 \\
\hline Nettoertrag aus Finanzgeschäften & 7.917 & & 7.917 & & 7.917 \\
\hline Sonstige betriebl. Erträge/Aufwendungen & 1.388 & & 1.388 & & 1.388 \\
\hline Ergebnis vor Bewertung & 59.537 & & 69.891 & & 71.101 \\
\hline $\begin{array}{l}\text { Risikovorsorge im Kreditgeschäft und } \\
\text { Bewertungsergebnis aus bestimmten } \\
\text { Wertpapieren und Beteiligungen }\end{array}$ & -13.353 & & -13.353 & & -13.353 \\
\hline Betriebsergebnis & 46.184 & & 56.538 & & 57.748 \\
\hline Neutrales Ergebnis und Ertragstevern & -8.832 & & -8.832 & & -8.832 \\
\hline Jahresüberschuss & 37.352 & & 47.706 & & 48.916 \\
\hline Cost/Income Ratio (CIR) & $49 \%$ & & $40 \%$ & & $39 \%$ \\
\hline
\end{tabular}

Fremdvergabe der Wertpapierabwicklung mit Ausnahme von Vertrieb und Kundenreporting.

$\mathrm{Zu}$ jedem Modell hat ein existierender Dienstleister ein Angebot abgegeben. Vorgegeben waren Ist-Darstellungen zu Geschäfts-, Prozess- und IS-Modell für den Wertpapierbereich sowie ein Mengengerüst der Modellbank (u. a. 120’000 Depots, Transaktionsmengen je Bankprodukt für In- und Ausland). Qualitative und quantitative Bewertung (s. Tabelle 5) führen zur Empfehlung eines selektiven Outsourcings des Auftragshandels (Modell 1). In gleicher Weise ließe sich aus Sicht der Gesamtbank ein übergeordnetes Sourcing-Modell bewerten, das sowohl aus dem Sourcing-Modell des Wertpapierbereiches als auch jenem zur Kreditabwicklung und zur Kunden-, Kontound Depotführung besteht.

\section{Fazit und Ausblick}

Die Unternehmensvernetzung ist heute in vielen Branchen, auch bei den traditionell konservativ agierenden und vertikal integrierten Banken in Form innovativer Inund Outsourcing-Strategien zu finden. Um die ökonomische Sinnhaftigkeit dieser häufig politisch dominierten Entscheidungen systematisch zu ermitteln, liefert das strategische Controlling eine wertvolle Unterstützung. Dies betrifft vor allem Instrumente zur Bewertung und zum systematischen Vergleich verschiedener Handlungsalternativen auf Basis von branchenbezogenem und möglichst konkretem Wissen zu Prozessen, Bankprodukten und Bewertungskriterien. Dieser Beitrag hat gezeigt, dass die klassischen Instrumente des strategischen Controllings zu erweitern sind. Dies sind einerseits die Kombination der klassischen quantitativen und qualitativen Bewertungsansätze sowie andererseits die Kopplung zu Ansätzen des Prozessmanagement. Letztlich setzt eine umfassende und realistische Bewertung eine (konsistente) Modellierung bezüglich Geschäfts-, Prozess- sowie IS-Modell voraus und kann einzig zu einer situativen Eignung von Sourcing-Modellen, nicht aber zu einer pauschalen Forderung nach maxima- 
Tabelle 5: Sourcing-Modelle bei der Modellbank (Ausschnitt aus Gesamtbewertung)

\begin{tabular}{|c|c|c|c|}
\hline & Sourcing-Modell 1 & Sourcing-Modell 2 & Sourcing-Modell 3 \\
\hline Prozessschritte & Auftragshandel & Auftragsverarbeitung & $\begin{array}{l}\text { Auffragserfassung, } \\
\text {-prüfung, -freigabe, } \\
\text {-handel, -verarbeitung }\end{array}$ \\
\hline \multicolumn{4}{|l|}{ Transaktionsneutrale Kosten } \\
\hline Positions-/Depotpostengebühr & - & $\begin{array}{l}1.5 \text { CHF je Position } \\
\text { und Quartal }\end{array}$ & $\begin{array}{l}1.5 \text { CHF je Position } \\
\text { und Quartal }\end{array}$ \\
\hline Verwahrungspreis Depot & $\begin{array}{c}0,02 \% \text { vom } \\
\text { Depotvolumen p. a. }\end{array}$ & $\begin{array}{c}0,02 \% \text { vom } \\
\text { Depotvolumen p. a. }\end{array}$ & $\begin{array}{c}0,02 \% \text { vom } \\
\text { Depotvolumen p. a. }\end{array}$ \\
\hline Managed Depots & - & - & $\begin{array}{c}0,1 \% \text { vom } \\
\text { Depotvolumen p. a. }\end{array}$ \\
\hline \multicolumn{4}{|l|}{ Transaktionsbezogene Kosten } \\
\hline Inland & $4 \mathrm{CHF}$ & $6,50 \mathrm{CHF}$ & $7 \mathrm{CHF}$ \\
\hline Ausland & $7 \mathrm{CHF}$ & $11 \mathrm{CHF}$ & $14 \mathrm{CHF}$ \\
\hline Derivate & $12 \mathrm{CHF}$ & $13 \mathrm{CHF}$ & $16,50 \mathrm{CHF}$ \\
\hline \multicolumn{4}{|l|}{ Betriebskosten } \\
\hline Fixer Betrag (Jahr). & $15 \%$ von Transformation & - & - \\
\hline Sourcing-Management & 20 PT р.а. & 60 PT р.а. & 60 PT р.а. \\
\hline Projektdauer (Monate) & 3 Monate & 9 - 10 Monate & 12 Monate \\
\hline Projektkosten & $60^{\prime} 000 \mathrm{CHF}$ & 1,8 Mio. CHF & 2,3 Mio. CHF \\
\hline Interne Aufwände & 100 PT & 1100 PT & 1400 PT \\
\hline \multicolumn{4}{|l|}{ Bewertung } \\
\hline Gesamtnutzwert & $\begin{array}{l}\text { 69,5\%, } 2 \text { negative } \\
\text { Abweichungen }\end{array}$ & $\begin{array}{l}\text { 63,6\%, } 6 \text { negative } \\
\text { Abweichungen }\end{array}$ & $\begin{array}{c}\text { 61,1\%, } 6 \text { negative } \\
\text { Abweichungen }\end{array}$ \\
\hline Barwert & $8^{\prime} 050 \mathrm{CHF}$ & $5^{\prime} 580 \mathrm{CHF}$ & 2'385 CHF \\
\hline Payback & 1 Jahr & 3 Jahre & 3 Jahre \\
\hline
\end{tabular}

lem Outsourcing führen. So wurde am Beispiel der beschriebenen Modellbank nicht das umfassende Sourcing-Modell 3, sondern ein selektives Outsourcing der spezifischen Aktivität Auftragshandel gewählt. Gemeinsam mit der Ausdifferenzierung der Branchenstruktur im Bankenbereich ist absehbar, dass derartige Einzelleistungen auf einer nächsten Wertschöpfungsstufe mit weiteren Leistungen verbunden werden und ein Anbieter die Abwicklung von Finanztransaktionen als Systemlösung ausführt.

Daraus ergeben sich drei unmittelbare Richtungen zur Weiterentwicklung des dargestellten Bewertungsmodells. Zunächst können sich mit der Bildung weiterer sektorspezifischer Modellbanken, also für Sparkassen, Genossenschaftsbanken und Grossbanken, Eignungspro- file für einzelne Sourcing-Modelle zur vereinfachten Vorselektion ableiten lassen. In einem weiteren Schritt könnten sowohl die Modellbanken als auch die Sourcing-Modelle in einem Modellierungstool bezüglich Geschäfts-, Prozessund IS-Modell abgebildet werden. Ein erster Prototyp auf Basis von MS Visio und der Erweiterung Semtalk liegt bereits vor. Zwar steht eine Integration des Bewertungsmodells in das Prozessmodellierungstool noch aus, die Aufnahme von Bewertungsmetriken in diese Tools ist aber noch ein Forschungsgegenstand. Schließlich erfordern Entwicklungen wie die Kaskadierung von Leistungsanbietern in der Bankenbranche iterative Bewertungen. Beispielsweise können durch die übergreifende Betrachtung von Einzelservices Abhängigkeiten und Synergien entstehen, die eine zu granulare Analyse vernachlässigt. In jedem Falle zeigt das Beispiel der Banken auch, dass diese eine kostenorientierte Sourcing-Entscheidung zunehmend in Richtung des „Strategic Sourcing“ überwinden. Das strategische Controlling als Versachlicher von Bauchentscheidungen dürfte dabei an Bedeutung gewinnen.

\section{Danksagung}

Die Autoren danken den Partnerunternehmen des Kompetenzzentrums „Sourcing in der Finanzindustrie“ (sourcing. iwi.unisg.ch) für ihre engagierte Mitarbeit und ihre finanzielle Unterstützung.

\section{Literatur}

AHr, H./KIPKER, I.: Kernkompetenzstrategien in Banken, in: SOKOLOVSKY, Z./LÖSCHENKOHL, S. (Hrsg.): Handbuch Industrialisierung der Finanzwirtschaft, Wiesbaden, 2005, S. $263-272$.

AMberG, M./Graf, L./WIENER, M.: Modelle für die Outsourcing-Entscheidung von Softwareentwicklungsprojekten, Nürnberg, 2005.

Beimborn, D./Franke, J./Weitzel, T.: The Role of Experience for Outsourcing Evaluation, in: Wirtschaftsinformatik, 47. Jg. (2005), Heft 6, S. $431-440$.

BERNET, B.: Aspekte produktionstopologischer Entscheidungen in Banken, in: GEIGER, H./SPREMANN, K. (Hrsg.): Banktopologie, Bern, 1998, S. $27-55$.

BRUCH, H.: Outsourcing, Konzepte und Strategien, Chancen und Risiken, Wiesbaden, 2000.

Corsten, D. S., Gabriel, C.: Supply Chain Management erfolgreich umsetzen: Grundlagen, Realisierung und Fallstudien, Berlin etc., 2004.

Dang, O./LaU, V.: The Swiss Opportunity: A Market Study on Outsourcing Solutions for Swiss Private Banks, London Business School, London, 2006.

DiCHTL, E.: Orientierungspunkte für die Festlegung der Fertigungstiefe, in: Wirtschaftswissenschaftliches Studium, (1991), Heft 2, S. 54 - 59. FALKenBerG, F./MÜller, R./BÖNSCH, J.: Wertschöpfungsmodelle der Zukunft - Banken und Provider 2010, Universität St.Gallen, Arbeitsbericht BE HSG/CC Sourcing/10, 2006.

FRITZ, W.: Marketing - ein Schlüsselfaktor des Unternehmenserfolges? Eine kritische Analyse vor dem Hintergrund der empirischen Erfolgsfaktorenforschung, in: Marketing ZFP, 12. Jg. (1990), Heft 2, S. 91 - 110.

Gottfredson, M./Puryear, R./Phillips, S.: Strategic Sourcing: From Periphery to the Core, in: Harvard Business Review, 83. Jg. (2005), Heft 1, S. $132-139$. 
Hagel, J.I./Singer, M.: Unbundling the Corporation, in: Harvard Business Review, 77. Jg. (1999), Heft 2, S. $133-141$.

HESs, T.: Netzwerkcontrolling: Instrumente und ihre Werkzeugunterstützung, Wiesbaden, 2002. IHDE, G.B.: Die relative Betriebstiefe als strategischer Erfolgsfaktor, in: Zeitschrift für Betriebswirtschaft, 58. Jg. (1988), Heft 1, S. $13-23$.

IMG: Sourcing-Markt Schweiz: Situation heute und zukünftige Entwicklungen, The Information Management Group, St.Gallen, 2004.

JeHLE, E.: Probleme und Lösungsmöglichkeiten bei der Steuerung von Unternehmensnetzwerken durch das strategische Controlling, in: Controlling, (2003), Heft 7/8, S. 379 - 387.

KeIL, P.: Principal Agent Theory and its Application to Analyze Outsourcing of Software Development, in: ACM SIGSOFT Software Engineering Notes, 40. Jg. (2005), Heft 4, S. $1-5$.

Kipker, I./VeIL, M.: Controlling- und Steuerungsinstrumente im Transaction Banking, in: KIPKER, I./VeIL, M. (Hrsg.): Transaction Banking: Strategien, Organisation, Steuerungsinstrumente, Wiesbaden, 2003, S. 183 - 196.

KLAus, P./Krieger, W.: Gabler Lexikon Logistik: Management logistischer Netzwerke und Flüsse, 3. Aufl., Wiesbaden, 2004.

KleIN, R./SCHOLL, A.: Planung und Entscheidung: Konzepte, Modelle und Methoden einer modernen betriebswirtschaftlichen Entscheidungsanalyse, München, 2004.

LAMBERTI, H.-J.: Industrialisierung des Bankgeschäfts, in: Die Bank, (2004), Heft 6, S. 370 - 375. LAMmers, M.: Make, Buy or Share - Combining Resource Based View, Transaction Cost Economics and Production Economies to a Sourcing Framework, in: Wirtschaftsinformatik, 46. Jg. (2004), Heft 3, S. $204-212$.

Locher, C./Mehlau, J.: Der IBI-Kubus - Ein multikausales Entscheidungsmodell für das Outsourcing unter Berücksichtigung spezifischer Rahmenbedingungen in der Finanzdienstleistungsbranche, in: BIT - Banking and Information Technology, Jg. (2002), Heft 4, S. 9 - 20.

Lubich, R./AumER, T.: Outsourcing im WPService: Die Total Cost of Ownership sind entscheidend, in: KIPKER, I./VEIL, M. (Hrsg.): Transaction Banking, Strategien, Organisation, Steuerungsinstrumente, Wiesbaden, 2003, S. 47-60. MÖLLER, K.: Gestaltungsbeitrag der Neuen Institutionenökonomik für das Controlling, in: Gleich, R./Möller, K./SEIDENSCHWARZ, W./ STOI, R. (Hrsg.): Controllingfortschritte, München, 2002, S. $95-120$.

Nadig, L.: Prozesskostenrechnung in Theorie und Praxis, Zürich, 2000.

Österle, H.: Business Engineering: Prozess- und Systementwicklung, Band 1: Entwurfstechniken, 2. Aufl., Berlin etc., 1995.
PICOT, A.: Ein neuer Ansatz zur Gestaltung der Leistungstiefe, in: Schmalenbachs Zeitschrift für betriebswirtschaftliche Forschung, 43. Jg. (1991), Heft 4, S. $336-357$.

Picot, A./DieTL, H./FranCK, E.: Organisation: Eine ökonomische Perspektive, Stuttgart, 2002. POHLAND, S.: Globale Unternehmensarchitekturen: Methode zur Verteilung von Informationssystemen, Berlin, 2000.

SChILdHauer, T.: Methoden zur Bestimmung optimaler Leistungstiefe der Informations- und Kommunikationstechnik - am Beispiel des Unfallkrankenhauses Berlin, Diss. TU Berlin, 2001. SIMSCHEK, R.: Ansätze zur Bewertung im Financial Sourcing, unveröffentlichte Diplomarbeit Universität Hohenheim, Stuttgart 2005.

STAHL, H.-W.: Controlling - Theorie und Praxis einer effizienten Systemgestaltung, Wiesbaden, 1992.

Welch, J.A./NAYAK, P.R.: Strategic Sourcing: A Progressive Approach to the Make-or-buy Decision, in: Academy of Management Executive, 6. Jg. (1992), Heft 1, S. 23 - 31.

Wikipedia, Bewertung, http://de.wikipedia.org/ wiki/Bewertung, Abruf am 27.4.2006.

Williamson, O. E.: The Economic Institutions of Capitalism: Firms, Markets, Relational Contracting, New York/London, 1985.
State-of-the-Art der Unternehmensbewertung

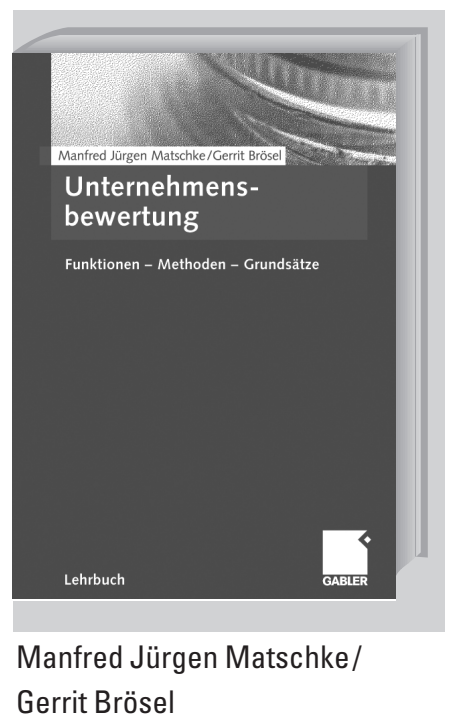

\section{Unternehmensbewertung}

Funktionen - Methoden - Grundsätze 2005. XXXII, 713 S. Geb. EUR 44,90 ISBN 3-8349-0012-5

Inhalt: Grundlagen - Entscheidungsfunktion und Entscheidungswert Vermittlungsfunktion und Arbitriumwert-Argumentationsfunktion und Argumentationswert-Grundsätze der Unternehmensbewertung

Die Autoren: Prof. Dr. Manfred Jürgen Matschke ist Inhaber des Lehrstuhls für Allgemeine Betriebswirtschaftslehre und Betriebliche Finanzwirtschaft, insbesondere Unternehmensbewertung an der Ernst-Moritz-Arndt-Universität Greifswald.

Dr. Gerrit Brösel ist Habilitand und Wissenschaftlicher Assistent am Fachgebiet Rechnungswesen/Controlling der Technischen Universität IImenau. Siehe auch: www.konvergenz-management.com.

Änderungen vorbehalten

Erhältlich im Buchhandel oder beim Verlag.

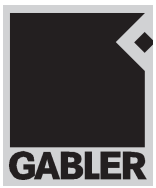

